\title{
Conceptual Modeling for Crawl Swimmers' Hand Using Control-Learning: A Simulation Approach
}

\author{
Sara Sheikh ${ }^{1}$, Majid Pouladian ${ }^{2}$, Reza Sarang ${ }^{3}$ \\ ${ }^{1}$ Biomechanics Department, Biomedical Engineering School, Central Tehran branch, Islamic Azad University \\ Tehran, Iran \\ sarah.sheikh7411@gmail.com \\ ${ }^{2}$ Bioelectric Department, School of Engineering, Science and research branch, Islamic Azad University \\ Tehran, Iran \\ pouladian@srbiau.ac.ir \\ ${ }^{3}$ Sports Engineering Department, Science and research branch, Islamic Azad University \\ Tehran, Iran \\ r-Sarang@srbiau.ac.ir
}

\begin{abstract}
We introduce a conceptual learning model of the optimal crawl swimmers' arm angle for the first time. We utilize a closed-loop control model and a neural network to develop this model. The presented model helps the professional swimmers to find their best possible arm's angle considering their characteristics. The developed model can also be used to predict and optimize professional swimmers' most important factor (arm angle) that influence their result in crawl swimming competitions.
\end{abstract}

Keywords: Homomorphic model, Crawl swimming, Musculoskeletal control, Neural network.

\section{Introduction}

The analysis of swimming movements is of particular importance in professional swimming competition as a fraction of second is decisive for swimmers. By using neuromuscular control and neural network, we get a more accurate learning model for swimmers. In this article, we take a positive step towards learning models for swimmers and take conceptual criteria instead of sensory criteria for swimmer instructors. Swimming instructors require a method for converting descriptive criteria to numerical criteria to evaluate swimmers at different times. We also show a homomorphic neurological and learning control model to formulate swimmers' hand movements.

\section{Literature Review}

The modeling procedures are divided into three main categories: 1) homomorphic or analytical method 2) Empirical method; and 3) Hybrid method. In the homomorphic or analytical method, the relationships between the components and their values are known. The second method is repetitive where information about the relationships and components of the system is unknown or we do not want to use them. The third method is a combination of the two methods above. In this way, we do not have complete information about the entire system, but we have some information about its structure. Mathematical modeling of the physiological system is usually composed of differential equations for describing the dynamics of the system. Using differential equations and simulating them with a computer requires numerical estimates of the parameters and the initial values of the variables. Many physiological models are based on physical laws, and they aim to obtain models that reflect the model's behavioral and qualitative issues. These physiological models are derived using advanced mathematics where the model parameters are estimated based on empirical and experimental measurements. These models require a computer and numerical analysis software for simulation purposes [1]. 
Hill's Model: The total force of a live muscle is divided into two active and passive sections. The active part is the result of muscle contraction and the inactive portion is due to the viscoelastic properties. Hill's muscle model consists of three parts including a contraction component, elastic part, and elastic series. This is one of the most famous models that explain the vibrating behavior and force produced in the muscles. The arrangement of the Hill model components is illustrated in the following figure.

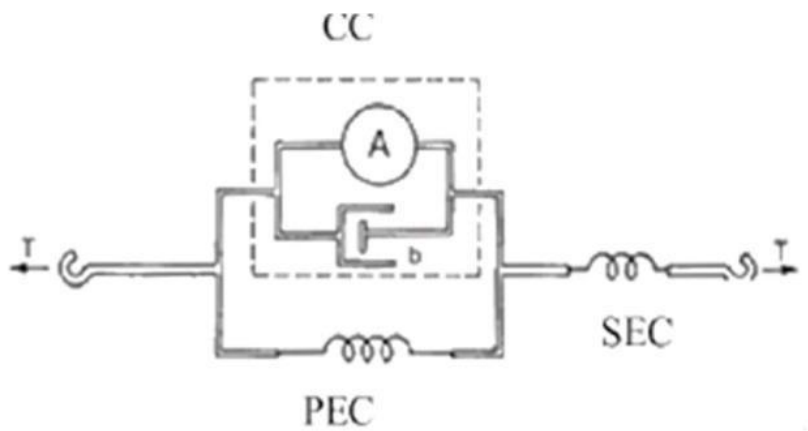

Fig. 1: Components of Hill's Model.

Hill's model is a mathematical and phenomenological model where the contraction component is considered as the action of actin and myosin in the formation of transverse bridges and the production of contractile force. The parallel elastic component represents the behavior of the fascia and other connective tissues that surround the contractions, and the elastic component of the series represents the behavioral tendencies of the tendons and myofilaments. Research has shown that the force produced in the muscle in the isometric condition is more than the force produced at shortening. The weakening mechanism of the transverse bridges along with muscle contraction as well as the viscosity of the muscle are the reasons for this. Accordingly, the contraction component in the Hill model consists of a damper (b) and an appropriate function (A) for expressing muscle contraction. [2]

KAWATO's Model: pointed out that the feedback-error-learning approach can be demonstrated mathematically using a Newtonian approach to functional space. In advanced mammals, like humans, learning under control is the most important part of the learning center. In almost all cases, the trainer cannot directly show the correct driving instruction to the trainer but can show the correct direction of movement. Consider the neural networks that receive the desired motor pattern. The path received by various sensory systems is measured and compared with the desired motor pattern (our educational signal is our monitoring signal). If the trainer can show the difference between the real-life steering wheel and the actual driving command, different regulatory instructional rules can be used to teach the control networks.

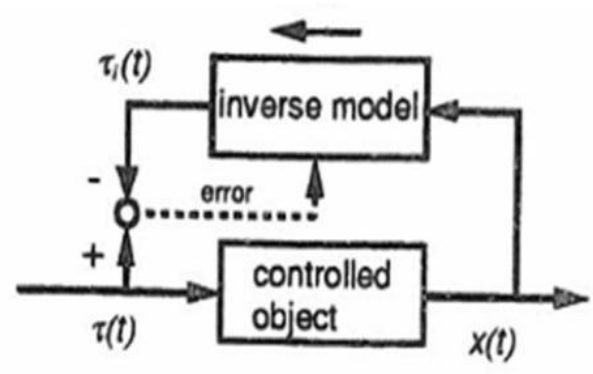

Fig. 2: Direct Reverse Modeling. 
The controlled object receives the torque input $\tau(\mathrm{t})$ and delivers the $\mathrm{x}(\mathrm{t})$ path as output. The inverse model for input and output direction in a controlled object is shown in Figure 2. This means taking the path as an input and delivering torque output. The error signal in Equation (1) is the difference between the actual torque and the estimated torque.

$$
S(t)=\tau(t)-\tau_{i}(t)
$$

This approach is known as a reverse direct inverse model. Figure 3 illustrates the feedback training approach.

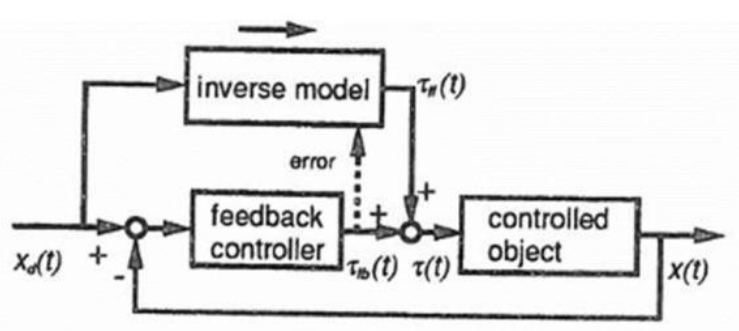

Fig. 3: feedback error learning.

The total torque $\tau(\mathrm{t})$ enters the controlled element of the sum of the feedback torque $\tau(\mathrm{t})$ and the torque ( ) obtained in the inverse model [3]. The reciprocal model receives the path and monitors the feedback torque ( )for the error signal. It is expected that the feedback signal, as the training continues, will go to zero. We refer to this process as a feedback error.

Comparative control of reference model: Model Adaptive Systems - Reference (MRAS) is one of the most important adaptive controllers. This system can be considered as a comparative servo system, in which the optimal performance is expressed in terms of the reference model that gives the command signal a desirable response. The block diagram of this system is shown in Figure 4. The system has common feedback, consisting of a process and a controller, and another loop that changes the controller's parameters. Parameters are changed based on the source code, which is the difference between the system output and the output of the reference model.

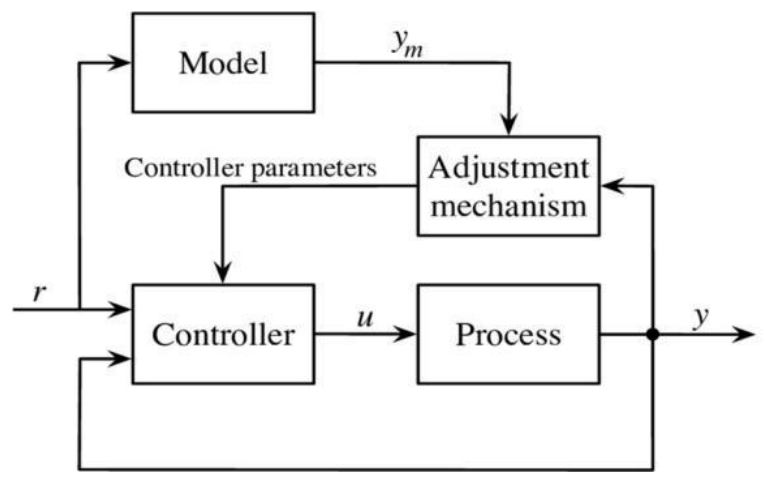

Fig. 4: Model-Reference Comparative System Block Map (MRAS).

In the MRAS system, the optimal behavior of the system is determined by a model, and the controller parameters are set based on an error, which is the difference between the outputs of the closed-loop system and the model [4-5]. Musculoskeletal models are important tools for studying muscle-driven movements. Active sensing in these models involves the production of signals to acquire sensory information [6-8]. 


\section{Problem Definition}

The nerve signal is sent from the upper centers (the brain) to the muscles of the hand by the spinal cord. The nervous signal is sent to the region of the sides of the elbow, arm, and muscles of the agonist and antagonist. This causes agonist muscle contraction and antagonist muscle release. At the site of the elbow, the force creates the desired torque in the swimmer's hand. Theta angle is the angle formed at the elbow between the forearm and the $\mathrm{arm}$. This is the angle in the chisel with a rhythmic motion. Usually, these changes range from 100 to 130 degrees. Ultimately, the swimmer's brain gains false feedback every time and compares the error with the optimal model that is obtained through training. As shown in Figure 5, this feedback goes up to the point where the theta error converges to zero with the ideal model.

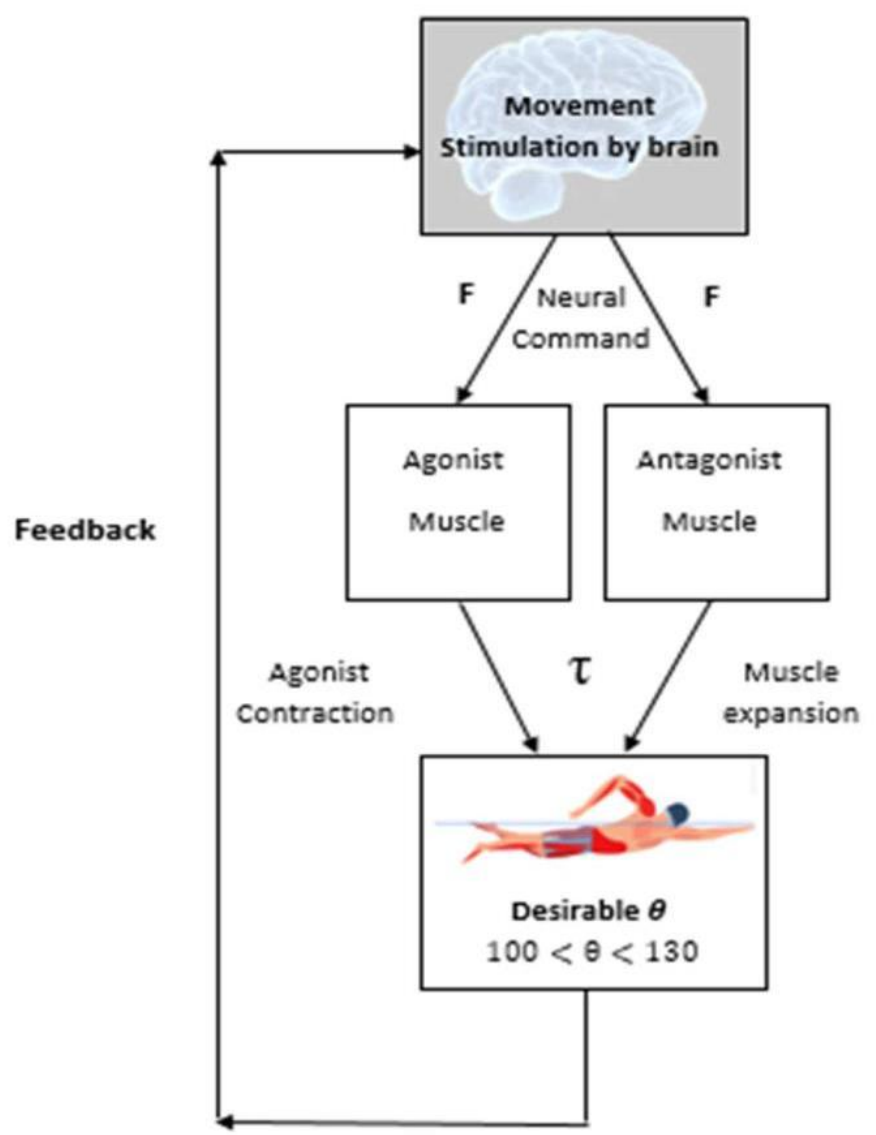

Fig. 5: Flowchart The pathway for transmitting the nerve signal from the brain to the elbow.

In swimming, the chest is more important than the swimmer's foot. Because the chest is progressing from the area of the hand. With that in mind, we focused on one hand. On one hand, the part that is important to us in this project is the elbow area. Nervous-muscular control was used to better understand neuromuscular coordination and how to send the steering wheel from the brain to the muscles in the elbow area. The Black Box's neural network is the job of adjusting the weights to learn the best hand angle.

A neural network can be used to build a learning model that can be applied to the brain during chest movement, especially the elbow angle. Using this software, we can do this modeling in the neural network. There are many factors involved in obtaining the desired angle of the elbow in the hand of the Crawl swimmer, here we refer to two of the most important inputs. We consider force and muscle length as input, and the command of contraction of the desired muscle to the target muscle (To give the signal a command to shrink the muscle). With the desired amount of muscle contraction, which is one of the muscles around the swimmer's elbow, the torque is created in the joint and the elbow of the swimmer can be bent to $\theta$ optimally. 
With this diverse data from real swimmers, this conceptual model can be developed. Here, the feedback from the muscle and the duct returns to the neural network to move the angle of inclination and the intensity of the contraction command to an optimum amount.

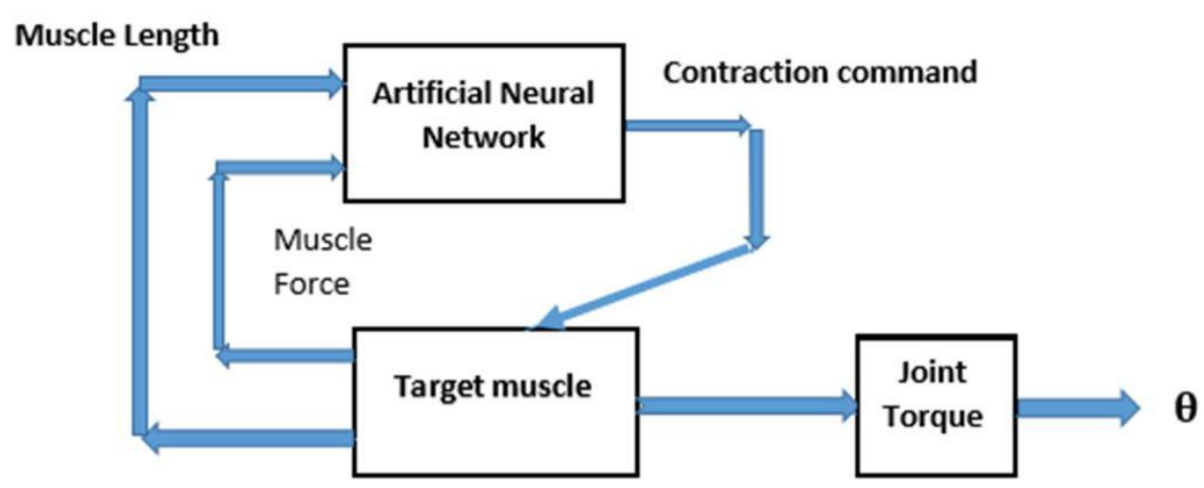

Fig. 6: Homomorphic model of a swimmer's muscle.

\section{Modeling Results}

In this article, the basis for learning in the brain of the beginner swimmer occurs under the supervision of the coach. For network inputs, we considered two inputs, including muscle length feedback and agonist muscle force feedback. The network output was determined by the command of the muscle contraction, or, in other words, the muscle strength at the site of the elbow. The range of elbows and muscles of the agonist and antagonist that helps bend the elbows is important for us in this article. The model we are looking for is a conceptual model, which can be derived from the neural network and control models and can determine the parameters of the neural network specific to each swimmer. Because at each moment the position of the hand and the elbow angle changes, our nervous system is variable with time and Dynamic, which should be checked with the rules of such networks. The movement of the signal to the muscles of the hand is shown in the following diagrams.

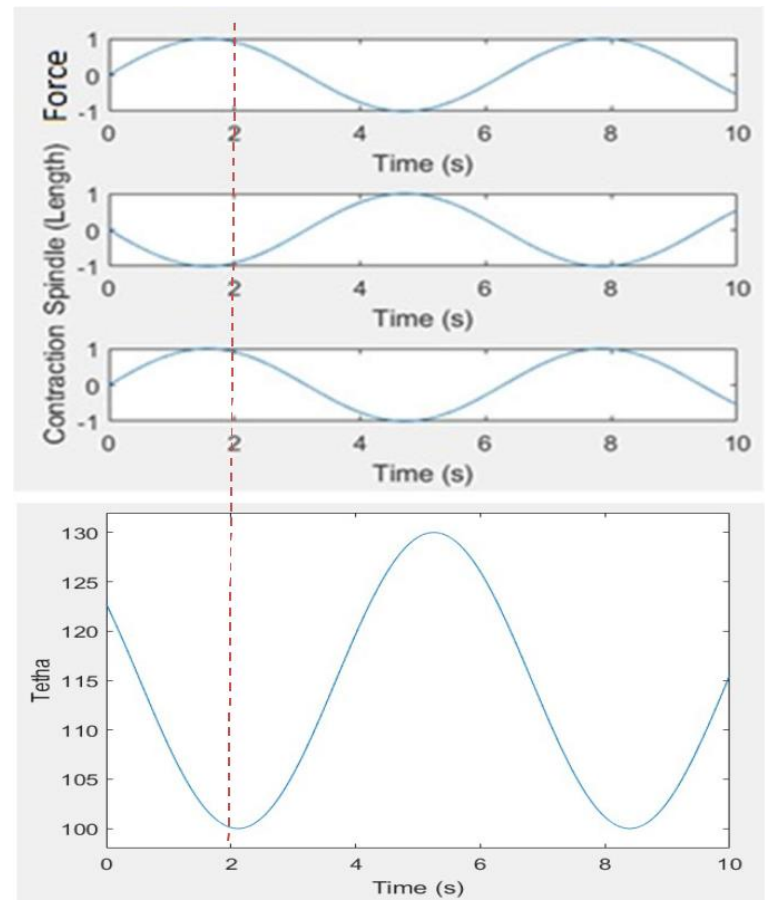

Fig. 7: Comparison of four angular factors, muscle contraction, muscle length, and force versus time. 
In this figure, the contraction outputs, duct length, muscle strength, and hand angle relative to time are shown. Graphs show that outputs are oscillating. For example, at the moment $t=2 \mathrm{~s}$, the contraction has a maximum value, that is, the contractile muscle. In this case, the duct is in its shortest position (there is a photo relationship with muscle contraction). At the same time, the force increases and leads to the bending of the elbow angle, which is $\theta$ optimal for us. Force and contraction have a direct relationship. The graph $\theta$ in time shows that the swimmer swings between the angles of 100 to 130, and at the instant $t=2 \mathrm{~s}$ the angle is reduced to 100 degrees.

\section{Conclusion and Recommendations}

We used homomorphic modeling to simulate the best angle for chest pains. Our presented neural network model is dynamic since the inputs and outputs are variable over time. Many inputs are involved in learning the data entered into the swimmer's brain. Therefore, we consider two of the most important inputs, changes in muscle length, and muscle strength (tendon). We presented the identification of linear variable-time systems using Feedforward's neural network [1-2]. We also showed the impact of weights changes on the output over time. Our goal was to show how learning and swimming skills are acquired in each swimmer. We suggest that, given availability, the electromyography device (EMG) acquires the swimmer muscle signals and, considering the intensity of the signals, examine the muscular weaknesses of the swimmer. This process can be continued until the swimmer coach has brought each swimmer closer to the desired path. The presented model can help the professional swimmers to find their best possible arm's angle considering their characteristics. The developed model can also be applied for predicting and optimizing professional swimmers' arm angle that significantly influences their result in crawl swimming competitions.

\section{References}

[1] Javadinejad. Ahmad, The Basis of Mathematics, Image Recovery in Radiology (Medicine), Ferdowsi University of Mashhad, 1996.

[2] Ashrostaghi. Mojtaba, Sadeghi. Heydar, Shirzad. Elham, "Review of the Concept of Stiffness in the Research on Mechanical Properties and Behavior of Human Body and Its Measurement Methods in Lower Extremity", Review Article, IRAN, Published Online: 2016.June.23.

[3] Kawato M, Feedback- Error- Learning Neural Network for Supervised Motor Learning, Cognitive Processes Department ATR Auditory and Visual Perception Research Laboratories, Kyoto, Elsevier Science Publishers B.V. (North- Holland), 1990.

[4] Osburn, P.V., H.P. Whitaker, and A. Kezer, 1961."New developments in the design of adaptive control system." Paper No. 61-39, February 1961, Inst. Aeronautical Sciences.

[5] Biswas, D., Arend, L. A., Stamper, S. A., Vágvölgyi, B. P., Fortune, E. S., \& Cowan, N. J. 2018. Closedloop control of active sensing movements regulates sensory slip. Current Biology, vol. 28, no. 24, pp. 40294036.

[6] O'Neill, M. C., Lee, L. F., Larson, S. G., Demes, B., Stern, J. T., \& Umberger, B. R. (2013). A threedimensional musculoskeletal model of the chimpanzee (Pan troglodytes) pelvis and hind limb. Journal of Experimental Biology, vol. 216, no. 19, pp. 3709-3723.

[7] Papadimitriou, K., Loupos, D., Tsalis, G., \& Manou, B. (2017). Effects of proprioceptive neuromuscular facilitation (PNF) on swimmers leg mobility and performance. Journal of Physical Education and Sport, vol. 17 , no. 2 , pp. 663.

[8] Cioroiu, S. G., \& Nechita, F. (2016). Swimming neuromuscular control. Ovidius University Annals, Series Physical Education and Sport/Science, Movement and Health, vol.16(2 SI), pp. 364-370. 